\title{
Altered temporal, but intact spatial, features of transient network dynamics in psychosis
}

\author{
Danhong Wang ${ }^{1} \cdot$ Xiaolong Peng $\mathbb{1}^{1,2} \cdot$ Andrea Pelletier-Baldelli, $i^{3,4}$ Natasza Orlov ${ }^{1} \cdot$ Amy Farabaugh $^{4}$. \\ Shahin Nasr ${ }^{1} \cdot$ Hamdi Eryilmaz ${ }^{4}$ Maurizio Fava ${ }^{4} \cdot$ Avram J. Holmes $\mathbb{D}^{5} \cdot$ Joshua L. Roffman ${ }^{1,4} \cdot$ Hesheng Liu ${ }^{1,6,7}$. \\ Daphne J. Holt $\mathbb{1}^{1,4}$
}

Received: 15 July 2019 / Revised: 9 October 2020 / Accepted: 2 December 2020 / Published online: 18 January 2021

(c) The Author(s) 2021. This article is published with open access

\begin{abstract}
Contemporary models of psychosis suggest that a continuum of severity of psychotic symptoms exists, with subthreshold psychotic experiences (PEs) potentially reflecting some genetic and environmental risk factors shared with clinical psychosis. Thus, identifying abnormalities in brain activity that manifest across this continuum can shed new light on the pathophysiology of psychosis. Here, we investigated the moment-to-moment engagement of brain networks ("states") in individuals with schizophrenia (SCZ) and PEs and identified features of these states that are associated with psychosisspectrum symptoms. Transient brain states were defined by clustering "single snapshots" of blood oxygen level-dependent images, based on spatial similarity of the images. We found that individuals with SCZ $(n=35)$ demonstrated reduced recruitment of three brain states compared to demographically matched healthy controls $(n=35)$. Of these three illnessrelated states, one specific state, involving primarily the visual and salience networks, also occurred at a lower rate in individuals with persistent PEs $(n=22)$, compared to demographically matched healthy youth $(n=22)$. Moreover, the occurrence rate of this marker brain state was negatively correlated with the severity of PEs $(r=-0.26, p=0.003, n=130)$. In contrast, the spatial map of this state appeared to be unaffected in the SCZ or PE groups. Thus, reduced engagement of a brain state involving the visual and salience networks was demonstrated across the psychosis continuum, suggesting that early disruptions of perceptual and affective function may underlie some of the core symptoms of the illness.
\end{abstract}

\section{Introduction}

Studies conducted over the past decade have revealed that schizophrenia (SCZ) is associated with disruption of the

Supplementary information The online version of this article (https:// doi.org/10.1038/s41380-020-00983-1) contains Supplementary material, which is available to authorized users.

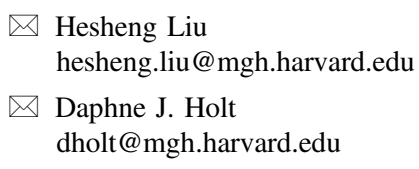

1 Athinoula A. Martinos Center for Biomedical Imaging, Department of Radiology, Massachusetts General Hospital, Harvard Medical School, Charlestown, MA, USA

2 Department of Radiology, Tongji Hospital, Tongji Medical College, Huazhong University of Science and Technology, Wuhan, China coordinated functioning of distributed brain networks [1-4]. These disruptions have been commonly identified by measuring fluctuations in low-frequency blood oxygen level-dependent (BOLD) signal in resting-state functional magnetic imaging (rs-fMRI) data [5-8]. Rs-fMRI data are typically examined using a "static" analysis, in which "functional connectivity" is estimated by measuring correlations of magnitudes of BOLD signals in distinct brain

3 University of North Carolina at Chapel Hill, Chapel Hill, NC, USA

4 Department of Psychiatry, Massachusetts General Hospital, Harvard Medical School, Boston, MA, USA

5 Department of Psychology, Yale University, New Haven, CT, USA

6 Department of Neuroscience, Medical University of South Carolina, Charleston, SC, USA

7 Beijing Institute for Brain Disorders, Capital Medical University, Beijing, China 
regions. Such static connectivity investigations have detected a wide range of alterations in functional connectivity in individuals with SCZ, within fronto-parietal, default mode, ventral attention, and somatosensory networks [9-11]. Although replicable differences in static connectivity between healthy and SCZ groups have been found [12], recent work has suggested that correlations of BOLD signals over minutes do not reflect the rapid changes in brain connectivity that may be most affected in psychotic illness [13-15]. Specifically, recent methodological advances have revealed that human brain activity is characterized by continuous, rapid shifts in activity across a large number of complex, varied networks, rather than a prolonged engagement of a small number of networks [16-19].

To leverage this new understanding of brain connectivity, investigations of the rapid shifts in BOLD signal correlations occurring across the brain have been recently conducted in SCZ. These studies have reported that individuals with SCZ show lower (or higher [20]) rates of switching among functional "states," reductions in the duration of time spent in cohesive states of regional co-activation, and decreased variation in states, compared to healthy subjects [21-25]. However, recent evidence suggests that findings of these "dynamic" studies that examine correlations of BOLD signals in short time windows are affected by unaddressed confounds related to sampling variability [26].

An alternative approach to measuring transient brain activation is to examine absolute co-activation levels of BOLD signal across the brain at each time point [27]. This method allows for a more direct investigation of the magnitude of activity of functional networks and their interactions, without relying on temporal correlations of BOLD signal in short time windows [26]. Here we used this approach to measure the configurations of functional networks, and the temporal and spatial properties of activity patterns of the brain, in individuals with SCZ and demographically matched healthy controls (HC). Critically, we used the same method in a second cohort of subjects: young adults with persistent subthreshold psychotic symptoms or what are commonly referred to as "psychotic experiences" (PEs) [28] and a group of demographically matched healthy youth (HY) without such symptoms. This second sample allowed us to determine whether any of the patterns observed in SCZ were also found in individuals with PEs, while avoiding the confounds that typically limit the interpretation of neuroimaging findings in SCZ, such as treatment with antipsychotic medication [29, 30].

Although, on average, only a small percentage of individuals with PEs will eventually develop clinical psychosis, the persistence of PEs over time substantially increases such risk (5-25 fold) [28, 31, 32]. Furthermore, recent evidence supports the model that PEs are on a biological continuum with clinical psychosis [33-37]. For example, recent studies have identified changes in brain structure and function in individuals with PEs that are similar to those observed in SCZ [38-42]. Similarities across findings in SCZ and PE groups suggest that such common findings may reflect manifestations of psychosis-related pathophysiology (and are less likely to result from effects of treatment or chronic illness in SCZ). Thus, in this study, we aimed to identify the changes in dynamic brain states that manifest across the psychosis spectrum, in both SCZ and PE groups.

\section{Materials and methods}

\section{Participants}

Three datasets were analyzed in this study. All participants provided written informed consent in accordance with the guidelines of the Institutional Review Boards of Partners Healthcare (Datasets I and III) and Harvard University (Dataset II). All images were collected on 3T Tim-Trio scanners (Siemens, Erlangen, Germany) with 12-channel phased-array head coils.

Dataset I included 35 patients with DSM-IV-diagnosed SCZ (age 41.80 $\pm 9.01 ; 8$ female) and 35 age, sex, and head motion-matched HC (age 39.49 \pm 10.43 ; 8 female, see Table 1) [43]. One to two resting-state (eyes open) scans (372 s/run) were obtained from each subject. Functional images were acquired using a gradient-echo echo-planar pulse sequence $(\mathrm{TR}=3000 \mathrm{~ms}, \mathrm{TE}=30 \mathrm{~ms}$, flip angle $=$ $85^{\circ}, 3 \times 3 \times 3 \mathrm{~mm}$ voxels, FOV $\left.=216\right)$. Structural data included a multi-echo T1-weighted magnetization-prepared gradient-echo image $(\mathrm{TR}=2200 \mathrm{~ms}, \mathrm{TI}=1100 \mathrm{~ms}$, $\mathrm{TE}=$ $1.54 \mathrm{~ms}$, flip angle $\left.=7^{\circ}, 1.2 \times 1.2 \times 1.2 \mathrm{~mm}\right)$. As expected, the SCZ group had a significantly lower mean IQ and years of education than the HC group.

Dataset II included 130 enrolled college students (age $19.5 \pm 1.3$; range 18-24; 91 female) with elevated scores on either a measure of depression (Beck Depression Inventory total score $>5$, or item \#9>0) or PEs (Peters et al. Delusions Inventory (PDI) total score $>7$ ). Among these subjects, 22 consistently showed moderately elevated PDI scores (total PDI score $>4$ over two time points 1 year apart) and were included in the PE group (age 19.23 \pm 1.07 ; 8 female). Twenty-two subjects who showed consistently low PDI scores $(<4$, at two time points 1 year apart) were included in the HY group $(19.27 \pm 1.28 ; 8$ female). The imaging protocol was identical to that of Dataset I. The PE and HY groups were matched in gender, age, years of education, and head motion (see Table 1).

Dataset III included 20 young healthy subjects (age $29.6 \pm 5.3$; range $24-40 ; 10$ female). The imaging protocol was identical to that of Datasets I and II. For this cohort only, each subject was scanned twice, once with eyes open and 
Table 1 Characteristics of the participant groups.

A. Demographically matched healthy controls $(\mathrm{CON})$ and individuals with schizophrenia (SCZ) (Dataset I)

\begin{tabular}{lccc}
\hline & CON $(n=35)$ & SCZ $(n=35)$ & $P$ value \\
\hline Age (years) & $39.49 \pm 10.43$ & $41.80 \pm 9.01$ & 0.32 \\
Gender & $77.14 \%$ male & $77.14 \%$ male & $>0.90$ \\
PANSS Total & & $72.00 \pm 12.72$ & \\
PANSS Positive & & $17.29 \pm 5.58$ & \\
Symptoms Subscale & & $20.35 \pm 4.84$ & \\
PANSS Negative & & $34.35 \pm 6.55$ & \\
Symptoms Subscale & & & \\
PANSS General & & $0.089 \pm 0.06$ & 0.30 \\
Symptoms Subscale & & & \\
Head motion (FD) & $0.076 \pm 0.05$ & $101.35 \pm 11.40$ & $<0.01$ \\
IQ & $111.76 \pm 9.49$ & $<.86 \pm 2.44$ & $<0.01$ \\
Years of education & $17.21 \pm 2.18$ & 12.86 & \\
\hline B. Demographically matched HY and PE subjects (Dataset II) & \\
\hline & HY $(n=22)$ & PE $(n=22)$ & $P$ value \\
\hline Age (years) & $19.27 \pm 1.28$ & $19.23 \pm 1.07$ & 0.90 \\
Gender & $63.64 \%$ male & $63.64 \%$ male & $>0.90$ \\
PDI total score & $2.09 \pm 1.48$ & $8.36 \pm 2.52$ & $<0.001$ \\
Head motion (FD) & $0.0453 \pm 0.023$ & $0.0423 \pm 0.016$ & 0.612 \\
Years of education & $13.23 \pm 1.31$ & $13.23 \pm 1.15$ & $>0.90$ \\
\hline CON & & & \\
\hline
\end{tabular}

CON healthy control group, SCZ schizophrenia group, PANSS Positive and Negative Syndrome Scale, $F D$ framewise displacement (in $\mathrm{mm}$ ), $H Y$ Healthy youth group, $P E$ psychotic experiences group, PDI Peters et al. Delusions Inventory.

once with eyes closed. These data were used to examine the effects of visual input on the outcome measures of this study.

See the Supplementary methods for additional details.

\section{Preprocessing}

Rs-fMRI were preprocessed using a previously described analysis pipeline [44], which included: (1) projecting the data from the subject's native space to FSL MNI152 space and then onto the FreeSurfer fsaverage6 surface space; (2) linear detrending and band-pass filtering $(0.01-0.08 \mathrm{~Hz})$; (3) regressing nuisance variables including the global signal, head motion parameters, and their first temporal derivatives [45]; (4) spatial smoothing with a $6 \mathrm{~mm}$ FWHM Gaussian curve; (5) down-sampling the BOLD fMRI data to a mesh of 642 vertices in each hemisphere; and (6) temporal normalization to the signal extracted from each vertex.

\section{Clustering-based single frame dynamic analysis}

We applied a $k$-means clustering algorithm to classify BOLD images into different groups based on their spatial similarity (using a cosine similarity metric) (Fig. 1), which has been previously employed to temporally decompose resting-state networks into multiple spontaneous coactivation patterns [27]. The cluster centroids were interpreted as unique "brain states." Each BOLD image was defined by its signal values across 1284 surface vertices. The preprocessed BOLD signals of all subjects were concatenated and temporally standardized. To improve computational efficiency, we used a principal component analysis to reduce the dimensionality of the feature space to 650 , which could explain $99 \%$ of the total variance in the rsfMRI. The optimal cluster number was selected according to the test-retest reliability of the resulted brain states in 100 young healthy subjects scanned using the same scanning protocol as for our data (see Supplementary Fig. S1). The solutions with $11,14,19,31,35$ clusters were relatively reliable. In this study, we focused on 19-states estimation for further analyses.

\section{Results}

\section{Rate of occurrence of specific brain states was reduced in individuals with $\mathrm{SCZ}$}

Dynamic brain states were defined by clustering the single volumes/images of fMRI data based on their spatial similarity (Fig. 1), i.e., images with similar spatial patterns were clustered together, representing a brain state. Here, the term "states" only refers to brain activity patterns rather than cognitive states. Nineteen "brain states" were derived from clustering the resting-state images from $\mathrm{SCZ}$ and $\mathrm{HC}$ groups (Supplementary Fig. S2). The probability of occurrence was not uniformly distributed across these states, i.e., some states appeared more often than others. The occurrence rate of each state was calculated as the percentage of frames assigned to the state.

Among the 19 states, we identified three states (States A, B, and C; see Supplementary Fig. S2) that demonstrated a reduced occurrence rate in SCZ, compared to $\mathrm{HC}(p<$ $0.001, p<0.001, p=0.012$, respectively, FDR-corrected for multiple comparisons, Fig. 2, see also Supplementary Fig. S3 for individual data points). No states showed an increased occurrence rate in SCZ compared to HC. Maps of these states were compared to seven canonical functional networks previously described [46] (see Supplementary Fig. S4). State A was characterized by strong activation in the fronto-parietal network (FPN) and deactivation in the motor-sensory network (MN). State B had the opposite pattern, i.e., deactivation in the FPN and strong activation in the MN. State $\mathrm{C}$ involved strong activation in the visual network (VN) and the salience network (SN), including the insula and angular gyrus, and deactivation in the default mode network. In the SCZ group, the occurrence rate of 

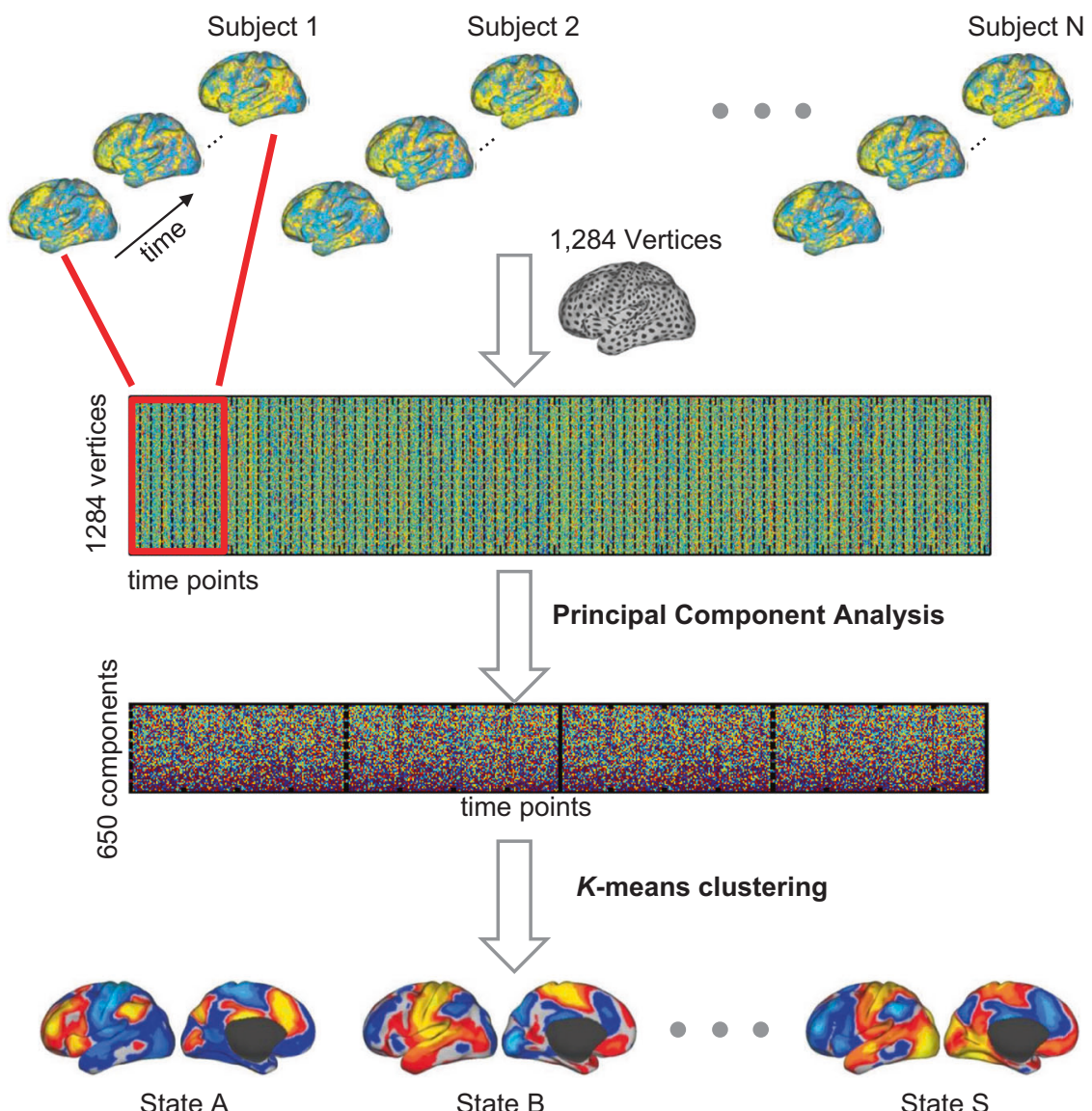

Fig. 1 Strategy for estimating brain network states from resting state fMRI. BOLD images were clustered into brain states according to their spatial patterns, i.e., images with similar spatial patterns were clustered together. Specifically, the preprocessed fMRI data of each individual subject was projected to the FreeSurfer fsaverage 3 surface space, which consisted of 1284 vertices in total. The data of different time points of each subject were aligned to construct a spatiotemporal matrix (indicated by the red rectangle). The data of all subjects were

these three states was uncorrelated with PANSS positive $(p>0.66)$ or negative $(p>0.32)$ symptom scores, or dose of antipsychotic medication $(p>0.17)$.

\section{The spatial distribution of brain states did not differ between SCZ and HC}

Although three states occurred less often in SCZ than in $\mathrm{HC}$, the spatial maps of these states appeared to be similar between the two groups (Spearman correlations between the state maps of two groups: $r=0.94,0.90,0.92$, respectively, see Supplementary Fig. S5). To further examine this similarity, we derived brain states from an independent dataset, the 100 healthy subjects that were used to determine the optimal cluster number (i.e., the test-retest dataset). Maps of States A, B, and C derived from the test-retest dataset resembled those derived from the $\mathrm{HC}$ and $\mathrm{SCZ}$ groups (Spearman correlations with the maps from HC: $r=0.94$, then concatenated and temporally standardized. Principal component analysis (PCA) was applied to the concatenated data matrix to reduce the dimensionality of data to 650 , which could explain more than $99 \%$ of the variance of the original data. The $k$-means clustering algorithm was then performed to classify the fMRI frames into a certain number of clusters (e.g., 19 clusters in this study). The fMRI frames assigned to the same cluster were averaged to generate the brain state map.

0.90, 0.84 for States A, B, and C, respectively; correlations with maps from SCZ: $r=0.91,0.81,0.77$, respectively). We then used the states derived from the test-retest dataset as cluster centers. In the $\mathrm{HC}$ and SCZ groups, we calculated spatial distance (Euclidean distance) from each frame to these cluster centers. For all three states, there was no difference in Euclidean distance from the frames to the cluster centers between the SCZ and HC groups (all $p>0.05$ ). These observations indicate that for each state, images from both groups are equally distributed around the cluster center defined in an independent dataset, confirming the spatial similarity of these images.

\section{Occurrence rate of State C was significantly reduced in PE}

To identify neural correlates of psychosis that are not confounded by medication effects or history of illness, we 


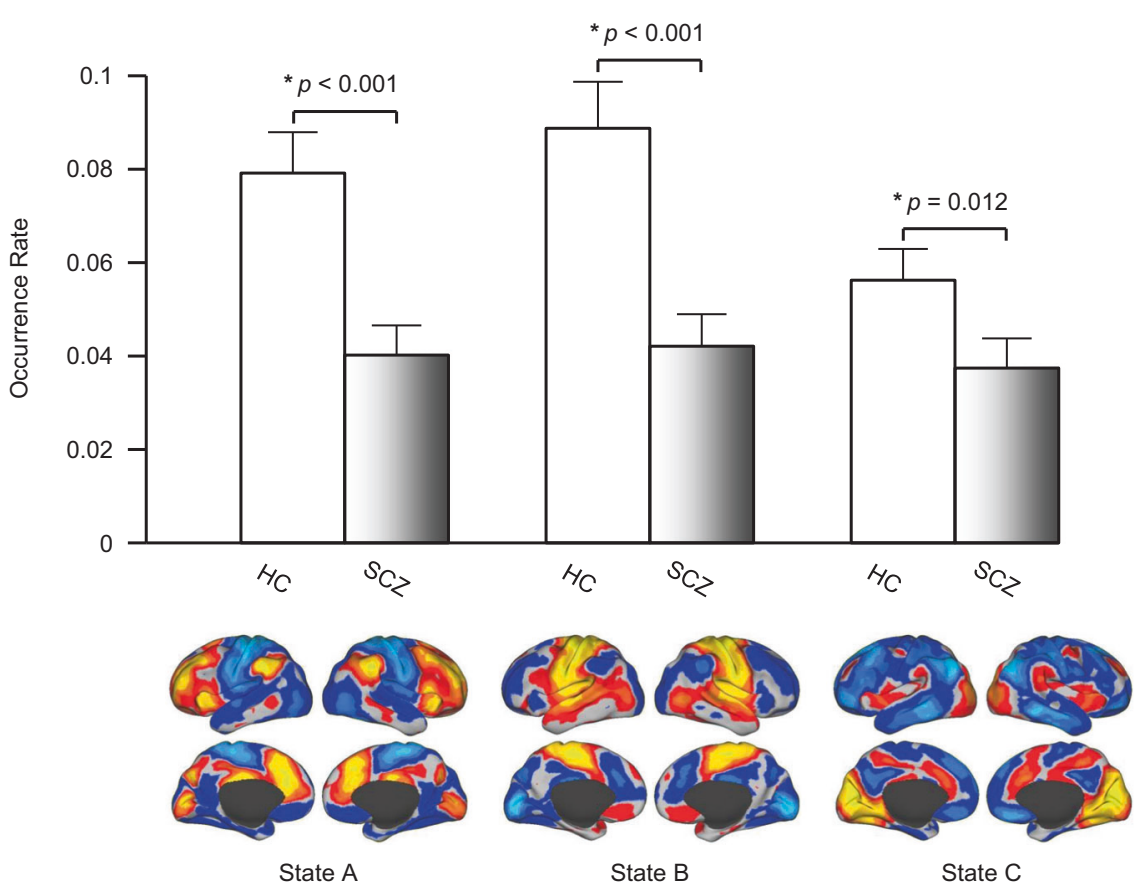

Fig. 2 The occurrence rate of three brain states was significantly reduced in patients with $S C Z$. Nineteen brain states were identified in the resting-state data from 35 patients with SCZ and 35 healthy controls. Three states demonstrated a significantly reduced rate of occurrence in the patients with SCZ compared to the controls $(p<$ $0.001, p<0.001, p=0.012$, two-tailed $t$-test, respectively). The maps of these brain states in the healthy controls are displayed in the lower panel. One state demonstrates strong activation in the fronto-parietal

also derived 19 states from the rs-fMRI data of the PE and the HY groups. The 19 states were identical to those described above. We found that only one state, State C, differed between the PE and HY groups. This state occurred at a significantly lower rate in PE than in HY $(p<0.001$, Fig. 3). However, the occurrence rates of State A and State B did not differ between the PE and HY groups $(p=0.16$, $p=0.20$, respectively).

The spatial map of State $\mathrm{C}$ also did not differ between the $\mathrm{PE}$ and HY groups (Spearman correlation: $r=0.910$, comparison of distance from frames to cluster center between the two groups: $p=0.79$ ). These observations indicated that, similar to our findings in SCZ, the spatial characteristics of these dynamic brain states are preserved in individuals with PEs.

\section{The occurrence rate of brain State $C$ was associated with the severity of PEs}

State C occurred at a lower rate in both SCZ and PE, compared to their controls, suggesting a specific association of this state to psychosis across a spectrum of severity. Thus, we examined whether the occurrence rate of State $\mathrm{C}$ could predict symptom severity in the full cohort of young control network but deactivation in sensorimotor areas. The second state demonstrates the opposite pattern. The third state involves strong activation in the visual cortex and the insula but deactivation in the default network. See also Supplementary Fig. S2 for the maps of the 19 clusters identified in this dataset and Supplementary Fig. S9 for the results using different cluster numbers (cluster number $=11,14$, $17,19,21)$.

adults that included the PE and HY groups, plus an additional 86 subjects with a low or intermediate level of PEs. Within this cohort $(n=130)$, the occurrence rate of State $\mathrm{C}$ showed a small correlation $(r=-0.26, p=0.003)$ with the number of PEs, as reflected by the subjects' total PDI score, indicating that the loss of State $\mathrm{C}$ is associated with the level of severity of PEs (explaining 6.7\% of the variance in PEs; Fig. 4).

\section{Static functional connectivity analyses}

To investigate how the loss of dynamic brain states is related to changes in conventional "static" functional connectivity, we selected three brain regions that were most activated in States A, B, and C and then computed functional connectivity between these selected seed regions and the rest of the brain (Supplementary Fig. S6). The static connectivity maps of these seed regions resembled the maps of dynamic brain states (all $p s<0.001$; see Supplementary Table S1). Moreover, compared to HC, the SCZ group showed reduced connectivity $(p<0.05$, FDR corrected, Supplementary Fig. S6) between the seeds and their connected regions, indicating that reduced occurrence of these states in patients with SCZ may be associated with reduced 
Fig. 3 A psychosis-linked "brain state" is mainly comprised of the visual cortical and salience networks. a Brain State C, which included activation of the visual cortex, insula, and dorsal anterior cingulate cortex, and deactivation in the default mode network, demonstrated a significantly reduced rate of occurrence in the SCZ and PE groups compared to controls $(p=0.012, p<0.001$, respectively, two-tailed $t$-test). $\mathbf{b}$ and $\mathbf{c}$ The spatial map of State $\mathrm{C}$ showed no differences between the SCZ and HC groups, or between the PE and the HY groups (Spearman spatial correlation: $r>0.90$ for both), indicating that spatial characteristics of this brain state are preserved (but its temporal characteristics are significantly altered) in the SCZ and PE groups.
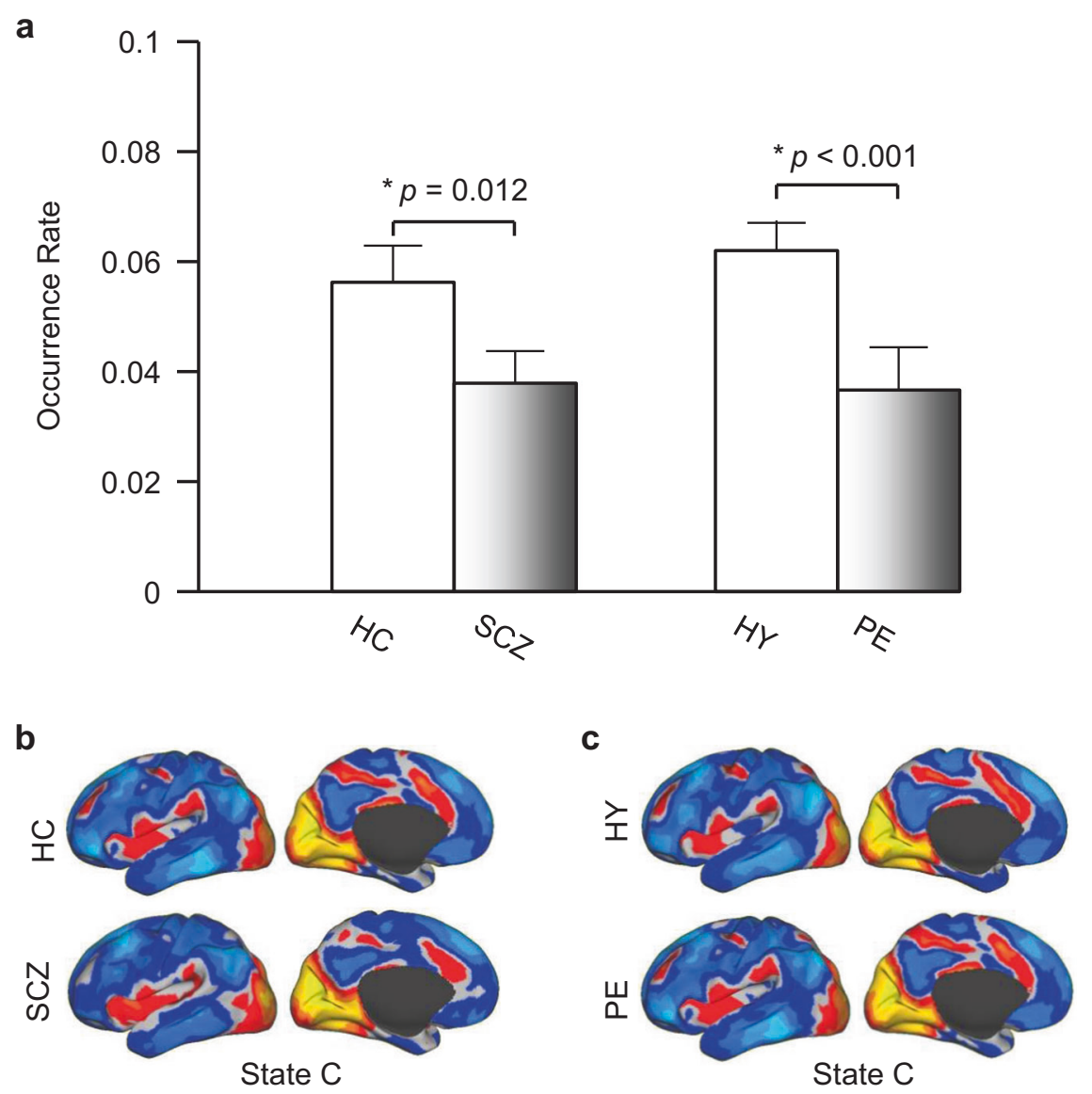

a

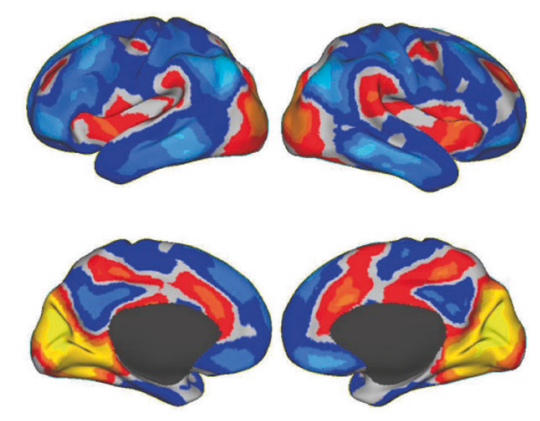

State C

Fig. 4 Occurrence rate of the psychosis-related state is associated with the severity of subthreshold psychotic symptoms. Within the full young adult cohort $(n=130)$, the occurrence rate of State $\mathrm{C}$ showed a significant correlation with PDI score $(r=-0.26, p=$ 0.003 , explaining $6.7 \%$ of the variance in PEs), indicating that the loss of State $\mathrm{C}$ is associated with the severity of subclinical psychotic

"static" functional connectivity between brain regions that are co-activated in these states. Similarly, compared to HY, the PE group also showed a trend of reduced connectivity (but did not reach significance after FDR correction) between a seed in the visual cortex and its connected

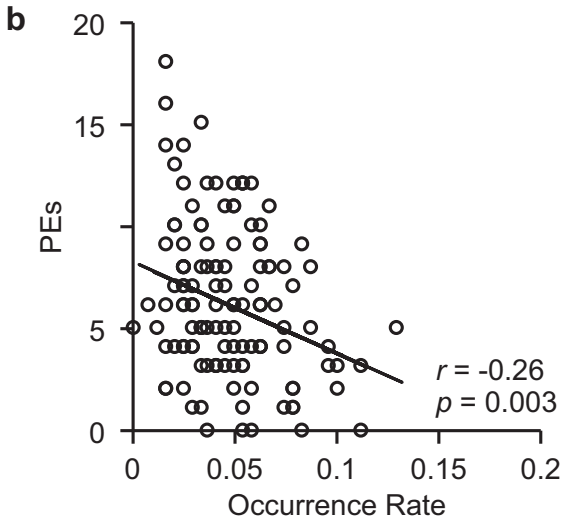

symptoms. A similar correlation between the loss of State C and psychotic symptom severity was not observed in the SCZ group, possibly due to the fact that all of the patients with SCZ enrolled in this study were being treated with antipsychotic medications at the time of data collection.

regions (Supplementary Fig. S6). This comparison of analysis approaches in the same cohort suggests that many of the prior reports of diminished functional connectivity in SCZ may be partially attributable to a reduction in the rate of recruitment of certain networks. Indeed, we further found 
that occurrence rates of States A, B, and C were correlated with static connectivity among the regions co-activated in these states (Supplementary Fig. S7).

\section{Potential confounding factors}

Analyses of data collected in healthy subjects scanned twice during rest, with eyes closed or open (Dataset III), indicated that our findings were not related to reductions in visual input in the SCZ or PE groups (see Supplementary results and Supplementary Fig. S8). In addition, we found that our results were not related to the selection of the number of clusters (see Supplementary results and Supplementary Fig. S9).

\section{Discussion}

In this study, measurement of the occurrence rate of "brain states," i.e., co-activated brain areas during one acquisition of rs-fMRI (collected over $3 \mathrm{~s}$ ), revealed three brain states that occurred at a lower rate in SCZ compared to HC. Of these three states, one also occurred at a diminished rate in individuals with persistent PEs. Moreover, the occurrence rate of this specific brain state was negatively correlated with the severity of PEs in a larger group of youth. In contrast, the spatial distribution of this state was unaffected in both the SCZ and PE groups.

\section{Reduced engagement of FPN, MN, and VN-SN in established psychotic illness}

Our results indicate that $\mathrm{SCZ}$ is associated with diminished recruitment of states involving the FPN, MN, and VN-SN. These results are consistent with and extend findings of prior neuroimaging studies of SCZ. Altered activation of the FPN in SCZ is a common finding of prior studies [10, 47] and has been linked to impairments in cognitive control, decision making, and the ability to initiate and persist in goal-directed behavior [48-52]. Similarly, altered sensorimotor and visual processes have been frequently reported in SCZ [53-55]. Prior studies have also revealed abnormalities in the SN in psychotic illness [56-59]. The current results suggest that a selective failure to appropriately recruit certain "brain states" that involve these networks may be present in SCZ.

\section{Reduced engagement of the VN-SN is also evident in individuals with persistent PEs}

A diminished occurrence rate of the $\mathrm{VN}-\mathrm{SN}$ state was also found in the PE group. Moreover, in the larger cohort of 130 youth (of which the PE and HY groups were subsets), reduced occurrence rate of this state was related to the severity of PEs. These results are consistent with prior evidence for a role of the SN and the insula in particular, a key node of the SN, in the pathophysiology of psychotic symptoms [60] (i.e., the replicated associations found between the activity or volume of the insula and psychotic symptoms in SCZ [61-67]). Although the mechanisms underlying these associations remain unclear, the current data taken together with evidence that the insula coordinates responses of widely distributed sensory and attentional networks and mediates transitions ("switching") between externally directed and introspective processing [56, 68-70] suggest that impairments in $\mathrm{SN}$-mediated transitions may occur in psychosis [56, 57]. Also, the insula is involved in selecting or prioritizing sensory information for the purpose of generating appropriate responses to that information [70-72]. Thus, our findings suggest that reduced recruitment of VN and SN may be associated with an impairment in this selection process, potentially contributing to the development of positive symptoms [57, 73].

\section{Model of progressive alterations in sensory, attentional, and higher-order neural systems in psychosis}

Our finding of selectively impaired engagement of the $\mathrm{SN}-\mathrm{VN}$ in the PE group, with the additional loss of function of the MN and FPN in SCZ, is suggestive of a progressive involvement of these networks in the pathophysiology underlying psychotic illness. We speculate that an initial disruption of sensory-salience processing at an early, subthreshold stage of illness may be followed by changes in the $\mathrm{MN}$ and FPN at later stages in the evolution of the illness. This model is consistent with studies showing that very early "psychosis risk" states are often characterized by the presence of mild sensory processing or cognitive changes that may involve inappropriately ascribing "salience" to incoming sensory information due to spontaneous neural signals that are independent of environmental stimuli (e.g., "basic symptoms" [74], "anomalous experiences" [75], or "aberrant salience" [76, 77]). For most, PEs are transient, often stress related and do not recur [78]. However, for a high-risk subset, these symptoms are distressing and persistent and, in some, associated with clinical progression. Progression may occur due to ongoing, persistent disruptions of sensory and salience processing, which ultimately interferes with cognitive and basic motor processes and function of the associated networks. Alternatively, the additional changes (the reduction in occurrence of States A and B) found in SCZ here, relative to those with PEs, could represent a long-term consequence of having the illness (e.g., the effects of chronic treatment with antipsychotic medication, a lack of employment or social activity). Further longitudinal work is required to test these competing 
models, by tracking activity of these brain states during sequential stages of illness. If such a model of progression is confirmed, low engagement of the $\mathrm{SN}-\mathrm{VN}$ could represent a marker of a very early, at-risk state.

\section{Psychotic experiences: associated risk for clinical psychosis and relationship to other prodromal states}

PEs have been studied primarily in non-help-seeking, general population samples with prevalence rates conservatively estimated at $\sim 7 \%$. The overall risk associated with PEs for transition to clinical psychosis is estimated at $\sim 8 \%[35,36]$, but some studies report higher transition rates (e.g., $25 \%$ [37]). The risk of transition depends on a number of factors such as the degree of persistence of the PEs, the presence of affective symptoms, and environmental factors [28, 79].

In contrast, the "clinical high risk" (CHR) category of the psychosis prodrome, which is also primarily defined by the presence of attenuated psychotic symptoms, includes only those who have become help-seeking and distressed, and show recent clinical deterioration [80]. Thus, the CHR state is associated with a much higher level of risk for developing psychotic illness than PEs [81]. Also, both PEs and the CHR state, while increasing risk for psychotic illness, are associated with an even greater likelihood of having or developing a non-psychotic affective or anxiety disorder $[28,82]$. Given the high levels of heterogeneity within these two overlapping categories and their association with transdiagnostic risk for neuropsychiatric illness overall, objective measures that can determine who are most vulnerable of those with these risk factors are clearly needed.

\section{Limitations}

A limitation of this study is that, in order to maximize feasibility and recruitment success, the members of the PE group were not clinically assessed for the presence of psychotic disorders. However, it is unlikely that any of the subjects of this study were acutely psychotic given that none were taking antipsychotic medications and all were fully enrolled in college at the time of the study. In addition, the cross-sectional nature of the study's design limits inferences regarding the relationship between the findings in the PE and SCZ groups; follow-up longitudinal studies can further test the model of illness progression suggested by these results.

It is not yet known whether the changes observed here in the occurrence of these transient brain states in the SCZ and $\mathrm{PE}$ groups reflect a relatively reversible impairment in neural responsiveness or synchrony, or a more enduring change in the integrity of the neurons within these networks or of their connections. However, the relationships between the occurrence of brain states and static connectivity suggest that changes in these brain states may be associated with enduring changes in functional coupling and network function. This hypothesis can be directly tested in future studies.

\section{Code availability}

Codes used to generate these results are available upon request (please contact $\mathrm{HL}$ ).

Acknowledgements DJH receives support from the Massachusetts General Hospital (MGH) Executive Committee on Research (the MGH Research Scholar Award), the Sidney R. Baer, Jr. Foundation, the Henry and Allison McCance Center for Brain Health, the Massachusetts Department of Mental Health Center of Excellence for Systemic and Psychosocial Research Services at MGH, and NIMH (RO1MH109562). HL receives support from the National Key Research and Development Program of China Grant No. 2016YFC1306303; the National Natural Science Foundation of China Grant No. 81790650, No. 81790652, and by NIH Grants R01NS091604, P50MH106435， P50DA046373， P20GM109040, R01DC017991, and R21MH121831. DW receives support from NIH Grant K01MH111802 and the NARSAD Young Investigator Award. This work was also supported by NIMH Grant K23MH084059 (JLR). The authors thank Dr. Randy L. Buckner for assistance with data collection.

\section{Compliance with ethical standards}

Conflict of interest HL is on the chief scientific advisory board for Neural Galaxy LLC and is listed as an inventor in issued patents on brain imaging that are unrelated to this work. JLR has received research support from Pamlab. MF's lifetime financial disclosures are listed here: http://mghcme.org/faculty/faculty-detail/maurizio_fava.

Publisher's note Springer Nature remains neutral with regard to jurisdictional claims in published maps and institutional affiliations.

Open Access This article is licensed under a Creative Commons Attribution 4.0 International License, which permits use, sharing, adaptation, distribution and reproduction in any medium or format, as long as you give appropriate credit to the original author(s) and the source, provide a link to the Creative Commons license, and indicate if changes were made. The images or other third party material in this article are included in the article's Creative Commons license, unless indicated otherwise in a credit line to the material. If material is not included in the article's Creative Commons license and your intended use is not permitted by statutory regulation or exceeds the permitted use, you will need to obtain permission directly from the copyright holder. To view a copy of this license, visit http://creativecommons. org/licenses/by/4.0/.

\section{References}

1. Friston KJ, Frith CD. Schizophrenia: a disconnection syndrome? Clin Neurosci. 1995;3:89-97.

2. McGuire PK, Frith CD. Disordered functional connectivity in schizophrenia. Psychol Med. 1996;26:663-7. 
3. Stephan KE, Friston KJ, Frith CD. Dysconnection in schizophrenia: from abnormal synaptic plasticity to failures of selfmonitoring. Schizophr Bull. 2009;35:509-27.

4. Pettersson-Yeo W, Allen P, Benetti S, McGuire P, Mechelli A. Dysconnectivity in schizophrenia: where are we now? Neurosci Biobehav Rev. 2011;35:1110-24.

5. Whitfield-Gabrieli S, Ford JM. Default mode network activity and connectivity in psychopathology. Annu Rev Clin Psychol. 2012;8: 49-76.

6. Whitfield-Gabrieli S, Thermenos HW, Milanovic S, Tsuang MT, Faraone SV, McCarley RW, et al. Hyperactivity and hyperconnectivity of the default network in schizophrenia and in firstdegree relatives of persons with schizophrenia. Proc Natl Acad Sci USA. 2009;106:1279-84.

7. Anticevic A, Cole MW, Repovs G, Murray JD, Brumbaugh MS, Winkler AM, et al. Characterizing thalamo-cortical disturbances in schizophrenia and bipolar illness. Cereb Cortex. 2014;24:3116-30.

8. Cole MW, Anticevic A, Repovs G, Barch D. Variable global dysconnectivity and individual differences in schizophrenia. Biol Psychiatry. 2011;70:43-50.

9. Mamah D, Barch DM, Repovs G. Resting state functional connectivity of five neural networks in bipolar disorder and schizophrenia. J Affect Disord. 2013;150:601-9.

10. Baker JT, Holmes AJ, Masters GA, Yeo BT, Krienen F, Buckner $\mathrm{RL}$, et al. Disruption of cortical association networks in schizophrenia and psychotic bipolar disorder. JAMA Psychiatry. 2014;71:109-18.

11. Woodward ND, Rogers B, Heckers S. Functional resting-state networks are differentially affected in schizophrenia. Schizophr Res. 2011;130:86-93.

12. Dong D, Wang Y, Chang X, Luo C, Yao D. Dysfunction of large-scale brain networks in schizophrenia: a meta-analysis of resting-state functional connectivity. Schizophr Bull. 2018;44:168-81.

13. Chang C, Glover GH. Time-frequency dynamics of resting-state brain connectivity measured with fMRI. Neuroimage. 2010;50: 81-98.

14. Cannon TD. How schizophrenia develops: cognitive and brain mechanisms underlying onset of psychosis. Trends Cogn Sci. 2015;19:744-56.

15. Keshavan MS, Giedd J, Lau JY, Lewis DA, Paus T. Changes in the adolescent brain and the pathophysiology of psychotic disorders. Lancet Psychiatry. 2014;1:549-58.

16. Bressler SL, Kelso JA. Cortical coordination dynamics and cognition. Trends Cogn Sci. 2001;5:26-36.

17. Deco G, Jirsa VK, McIntosh AR. Emerging concepts for the dynamical organization of resting-state activity in the brain. Nat Rev Neurosci. 2011;12:43-56.

18. Hutchison RM, Womelsdorf T, Allen EA, Bandettini PA, Calhoun VD, Corbetta M, et al. Dynamic functional connectivity: promise, issues, and interpretations. Neuroimage. 2013;80:360-78.

19. Bassett DS, Wymbs NF, Porter MA, Mucha PJ, Carlson JM, Grafton ST. Dynamic reconfiguration of human brain networks during learning. Proc Natl Acad Sci USA. 2011;108:7641-6.

20. Ma S, Calhoun VD, Phlypo R, Adali T. Dynamic changes of spatial functional network connectivity in healthy individuals and schizophrenia patients using independent vector analysis. Neuroimage. 2014;90:196-206.

21. Du Y, Pearlson GD, Yu Q, He H, Lin D, Sui J, et al. Interaction among subsystems within default mode network diminished in schizophrenia patients: a dynamic connectivity approach. Schizophr Res. 2016;170:55-65.

22. Miller RL, Yaesoubi M, Turner JA, Mathalon D, Preda A, Pearlson G, et al. Higher dimensional meta-state analysis reveals reduced resting fMRI connectivity dynamism in schizophrenia patients. PLoS One. 2016;11:e0149849.
23. Damaraju E, Allen EA, Belger A, Ford JM, McEwen S, Mathalon $\mathrm{DH}$, et al. Dynamic functional connectivity analysis reveals transient states of dysconnectivity in schizophrenia. Neuroimage Clin. 2014;5:298-308.

24. Yu Q, Erhardt EB, Sui J, Du Y, He H, Hjelm D, et al. Assessing dynamic brain graphs of time-varying connectivity in fMRI data: application to healthy controls and patients with schizophrenia. Neuroimage. 2015;107:345-55.

25. Reinen JM, Chen OY, Hutchison RM, Yeo BTT, Anderson KM, Sabuncu MR, et al. The human cortex possesses a reconfigurable dynamic network architecture that is disrupted in psychosis. Nat Commun. 2018;9:1157.

26. Laumann TO, Snyder AZ, Mitra A, Gordon EM, Gratton C, Adeyemo B, et al. On the stability of BOLD fMRI correlations. Cereb Cortex. 2017;27:4719-32.

27. Liu X, Duyn JH. Time-varying functional network information extracted from brief instances of spontaneous brain activity. Proc Natl Acad Sci USA. 2013;110:4392-7.

28. van Os J, Linscott RJ, Myin-Germeys I, Delespaul P, Krabbendam L. A systematic review and meta-analysis of the psychosis continuum: evidence for a psychosis proneness-persistenceimpairment model of psychotic disorder. Psychological Med. 2009;39:179-95.

29. Kirsch P, Ronshausen S, Mier D, Gallhofer B. The influence of antipsychotic treatment on brain reward system reactivity in schizophrenia patients. Pharmacopsychiatry. 2007;40:196-8.

30. Lui S, Li T, Deng W, Jiang L, Wu Q, Tang H, et al. Short-term effects of antipsychotic treatment on cerebral function in drugnaive first-episode schizophrenia revealed by "resting state" functional magnetic resonance imaging. Arch Gen Psychiatry. 2010;67:783-92.

31. Dominguez MD, Wichers M, Lieb R, Wittchen HU, van Os J. Evidence that onset of clinical psychosis is an outcome of progressively more persistent subclinical psychotic experiences: an 8year cohort study. Schizophr Bull. 2011;37:84-93.

32. van Os J, Linscott RJ. Introduction: the extended psychosis phenotype-relationship with schizophrenia and with ultrahigh risk status for psychosis. Schizophr Bull. 2012;38:227-30.

33. Guloksuz S, van Os J. The slow death of the concept of schizophrenia and the painful birth of the psychosis spectrum. Psychol Med. 2018;48:229-44.

34. Kelleher I, Cannon M. Psychotic-like experiences in the general population: characterizing a high-risk group for psychosis. Psychological medicine. 2011;41:1-6.

35. Hanssen M, Bak M, Bijl R, Vollebergh W, van Os J. The incidence and outcome of subclinical psychotic experiences in the general population. Br J Clin Psychol. 2005;44:181-91.

36. van Os J, Reininghaus U. Psychosis as a transdiagnostic and extended phenotype in the general population. World Psychiatry. 2016;15:118-24.

37. Poulton R, Caspi A, Moffitt TE, Cannon M, Murray R, Harrington H. Children's self-reported psychotic symptoms and adult schizophreniform disorder: a 15-year longitudinal study. Arch Gen Psychiatry. 2000;57:1053-8.

38. Drakesmith M, Caeyenberghs K, Dutt A, Zammit S, Evans CJ, Reichenberg A, et al. Schizophrenia-like topological changes in the structural connectome of individuals with subclinical psychotic experiences. Hum Brain Mapp. 2015;36:2629-43.

39. Orr JM, Turner JA, Mittal VA. Widespread brain dysconnectivity associated with psychotic-like experiences in the general population. Neuroimage Clin. 2014;4:343-51.

40. Satterthwaite TD, Vandekar SN, Wolf DH, Bassett DS, Ruparel $\mathrm{K}$, Shehzad Z, et al. Connectome-wide network analysis of youth with psychosis-spectrum symptoms. Mol Psychiatry. 2015;20: 1508-15. 
41. Satterthwaite TD, Wolf DH, Calkins ME, Vandekar SN, Erus G, Ruparel K, et al. Structural brain abnormalities in youth with psychosis spectrum symptoms. JAMA Psychiatry. 2016;73:515-24.

42. Wolf DH, Satterthwaite TD, Calkins ME, Ruparel K, Elliott MA, Hopson $\mathrm{RD}$, et al. Functional neuroimaging abnormalities in youth with psychosis spectrum symptoms. JAMA Psychiatry. 2015;72:456-65.

43. Eryilmaz H, Tanner AS, Ho NF, Nitenson AZ, Silverstein NJ, Petruzzi LJ, et al. Disrupted working memory circuitry in schizophrenia: disentangling fMRI markers of core pathology vs other aspects of impaired performance. Neuropsychopharmacology. 2016;41:2411-20.

44. Wang D, Buckner RL, Fox MD, Holt DJ, Holmes AJ, Stoecklein $\mathrm{S}$, et al. Parcellating cortical functional networks in individuals. Nat Neurosci. 2015;18:1853.

45. Power JD, Plitt M, Laumann TO, Martin A. Sources and implications of whole-brain fMRI signals in humans. Neuroimage. 2017;146:609-25.

46. Yeo BT, Krienen FM, Sepulcre J, Sabuncu MR, Lashkari D, Hollinshead M, et al. The organization of the human cerebral cortex estimated by intrinsic functional connectivity. J Neurophysiol. 2011;106:1125-65.

47. Tu PC, Lee YC, Chen YS, Li CT, Su TP. Schizophrenia and the brain's control network: aberrant within- and between-network connectivity of the frontoparietal network in schizophrenia. Schizophr Res. 2013;147:339-47.

48. Vincent JL, Kahn I, Snyder AZ, Raichle ME, Buckner RL. Evidence for a frontoparietal control system revealed by intrinsic functional connectivity. J Neurophysiol. 2008;100:3328-42.

49. Poppe AB, Barch DM, Carter CS, Gold JM, Ragland JD, Silverstein SM, et al. Reduced frontoparietal activity in schizophrenia is linked to a specific deficit in goal maintenance: a multisite functional imaging study. Schizophr Bull. 2016;42: $1149-57$.

50. Ray KL, Lesh TA, Howell AM, Salo TP, Ragland JD, MacDonald AW, et al. Functional network changes and cognitive control in schizophrenia. Neuroimage Clin. 2017;15:161-70.

51. Carter CS, Braver TS, Barch DM, Botvinick MM, Noll D, Cohen JD. Anterior cingulate cortex, error detection, and the online monitoring of performance. Science. 1998;280:747-9.

52. Weinberger DR, Gallhofer B. Cognitive function in schizophrenia. Int Clin Psychopharmacol. 1997;12:S29-36.

53. Kaufmann T, Skatun KC, Alnaes D, Doan NT, Duff EP, Tonnesen $\mathrm{S}$, et al. Disintegration of sensorimotor brain networks in schizophrenia. Schizophr Bull. 2015;41:1326-35.

54. Butler PD, Silverstein SM, Dakin SC. Visual perception and its impairment in schizophrenia. Biol Psychiatry. 2008;64:40-7.

55. Javitt DC. Sensory processing in schizophrenia: neither simple nor intact. Schizophr Bull. 2009;35:1059-64.

56. Uddin LQ. Salience processing and insular cortical function and dysfunction. Nat Rev Neurosci. 2015;16:55-61.

57. Palaniyappan L, Liddle PF. Does the salience network play a cardinal role in psychosis? An emerging hypothesis of insular dysfunction. J Psychiatry Neurosci. 2012;37:17-27.

58. Palaniyappan L, Simmonite M, White TP, Liddle EB, Liddle PF. Neural primacy of the salience processing system in schizophrenia. Neuron. 2013;79:814-28.

59. Manoliu A, Riedl V, Zherdin A, Muhlau M, Schwerthoffer D, Scherr M, et al. Aberrant dependence of default mode/ central executive network interactions on anterior insular salience network activity in schizophrenia. Schizophr Bull. 2014;40: 428-37.

60. Wylie KP, Tregellas JR. The role of the insula in schizophrenia. Schizophr Res. 2010;123:93-104.

61. Crespo-Facorro B, Kim J, Andreasen NC, O'Leary DS, Bockholt HJ, Magnotta V. Insular cortex abnormalities in schizophrenia: a structural magnetic resonance imaging study of first-episode patients. Schizophr Res. 2000;46:35-43.

62. Linnman C, Coombs G III, Goff DC, Holt DJ. Lack of insula reactivity to aversive stimuli in schizophrenia. Schizophr Res. 2013;143:150-7.

63. Pressler M, Nopoulos P, Ho BC, Andreasen NC. Insular cortex abnormalities in schizophrenia: relationship to symptoms and typical neuroleptic exposure. Biol Psychiatry. 2005;57:394-8.

64. Koutsouleris N, Gaser C, Jager M, Bottlender R, Frodl T, Holzinger $\mathrm{S}$, et al. Structural correlates of psychopathological symptom dimensions in schizophrenia: a voxel-based morphometric study. Neuroimage. 2008;39:1600-12.

65. Jardri R, Pouchet A, Pins D, Thomas P. Cortical activations during auditory verbal hallucinations in schizophrenia: a coordinate-based meta-analysis. Am J Psychiatry. 2011;168:73-81.

66. Shapleske J, Rossell SL, Chitnis XA, Suckling J, Simmons A, Bullmore ET, et al. A computational morphometric MRI study of schizophrenia: effects of hallucinations. Cereb Cortex. 2002;12: 1331-41.

67. Modinos G, Vercammen A, Mechelli A, Knegtering H, McGuire PK, Aleman A. Structural covariance in the hallucinating brain: a voxel-based morphometry study. J Psychiatry Neurosci. 2009;34: 465-9.

68. Corbetta M, Shulman GL. Control of goal-directed and stimulusdriven attention in the brain. Nat Rev Neurosci. 2002;3:201-15.

69. Corbetta M, Patel G, Shulman GL. The reorienting system of the human brain: from environment to theory of mind. Neuron. 2008; 58:306-24.

70. Menon V, Uddin LQ. Saliency, switching, attention and control: a network model of insula function. Brain Struct Funct. 2010;214: 655-67.

71. Uddin LQ, Supekar K, Amin H, Rykhlevskaia E, Nguyen DA, Greicius MD, et al. Dissociable connectivity within human angular gyrus and intraparietal sulcus: evidence from functional and structural connectivity. Cereb Cortex. 2010;20:2636-46.

72. Chang LJ, Yarkoni T, Khaw MW, Sanfey AG. Decoding the role of the insula in human cognition: functional parcellation and large-scale reverse inference. Cereb Cortex. 2013;23:739-49.

73. Powers AR, Mathys C, Corlett PR. Pavlovian conditioninginduced hallucinations result from overweighting of perceptual priors. Science. 2017;357:596-600.

74. Schultze-Lutter F. Subjective symptoms of schizophrenia in research and the clinic: the basic symptom concept. Schizophrenia Bull. 2009;35:5-8.

75. Maher BA. The relationship between delusions and hallucinations. Curr psychiatry Rep. 2006;8:179-83.

76. Kapur S. Psychosis as a state of aberrant salience: a framework linking biology, phenomenology, and pharmacology in schizophrenia. Am J Psychiatry. 2003;160:13-23.

77. Roiser JP, Howes OD, Chaddock CA, Joyce EM, McGuire P. Neural and behavioral correlates of aberrant salience in individuals at risk for psychosis. Schizophrenia Bull. 2013;39: $1328-36$.

78. Linscott R, Van Os J. An updated and conservative systematic review and meta-analysis of epidemiological evidence on psychotic experiences in children and adults: on the pathway from proneness to persistence to dimensional expression across mental disorders. Psychological Med. 2013;43:1133-49.

79. Dominguez M, Wichers M, Lieb R, Wittchen H-U, van Os J. Evidence that onset of clinical psychosis is an outcome of progressively more persistent subclinical psychotic experiences: an 8-year cohort study. Schizophrenia Bull. 2011;37: 84-93.

80. McGlashan TH, Walsh BC, Woods SW. Structured interview for psychosis-risk syndromes. New Haven, CT: Yale School of Medicine; 2001. 
81. Fusar-Poli P, Bonoldi I, Yung AR, Borgwardt S, Kempton MJ, Valmaggia L, et al. Predicting psychosis: meta-analysis of transition outcomes in individuals at high clinical risk. Arch Gen psychiatry. 2012;69:220-9.
82. Fusar-Poli P, Borgwardt S, Bechdolf A, Addington J, RiecherRössler A, Schultze-Lutter F, et al. The psychosis high-risk state: a comprehensive state-of-the-art review. JAMA Psychiatry. 2013;70:107-20. 